\title{
Estado nutricional como predictor de mortalidad en el adulto mayor con cáncer gástrico evaluado en un hospital de referencia
}

\author{
Edwin Cieza ${ }^{1,2,3, a}$, Teodoro J. Oscanoa ${ }^{1,2,3, b}$
}

RESUMEN

Objetivo: Evaluar el estado de nutrición como predictor de mortalidad en los adultos mayores con cáncer gástrico. Materiales y métodos: Estudio observacional, prospectivo y analítico. Se incluyeron 47 pacientes mayores de 60 años con diagnóstico de cáncer gástrico referidos al Servicio de Geriatría del Hospital Nacional Guillermo Almenara, de marzo del 2014 a octubre del 2015. Se evaluó con el índice de masa corporal (IMC) y el Mini Nutritional Assessment versión corta (MNA-SF).

Resultados: Se incluyeron 47 pacientes con diagnóstico de cáncer gástrico, 33 (70,2 \%) fueron de sexo masculino, la media de edad fue 75,52 (DE +/- 6,88) años para los varones y 77 (DE +/- 7,66) años para las mujeres. Al realizar el análisis de supervivencia, se encontró que la evaluación nutricional mediante el MNA-SF obtuvo significancia estadística $(p<0.05)$.

Conclusiones: Un estado nutricional deficiente es factor pronóstico en la mortalidad del cáncer gástrico en personas mayores.

Palabras claves: Estado nutricional; mortalidad; cáncer gástrico; personas mayores (Fuente: DeCS BIREME).

\section{Nutritional status as a predictor of mortality in elderly people with gastric cancer evaluated at a reference hospital}

\section{ABSTRACT}

Objective: To evaluate the nutritional status as a predictor of mortality in elderly people with gastric cancer.

Materials and methods: An observational, prospective and analytical study which included 47 patients over 60 years of age with a diagnosis of gastric cancer, referred to the geriatric department of the Hospital Nacional Guillermo Almenara from March 2014 to October 2015. The Body Mass Index (BMI) and the Mini Nutritional Assessment Short-Form (MNA-SF) were used to evaluate the patients.

Results: Forty-seven (47) patients with a diagnosis of gastric cancer were included: 33 (70.2 \%) were males, and the mean age was 75.52 (SD +/ - 6.88) years for males and 77 (SD + / - 7.66) years for women. When performing the survival analysis, it was found that the nutritional assessment using the MNA-SF obtained statistical significance ( $p<0.05)$.

Conclusions: In conclusion, A poor nutritional status is a prognostic factor of gastric cancer mortality in elderly people.

Keywords: Nutritional status; mortality; stomach neoplasms; aged (Source: MeSH NLM).

1. Hospital Nacional Guillermo Almenara Irigoyen. Lima, Perú.

2. Universidad Nacional Mayor de San Marcos, Facultad de Medicina, Departamento de Ciencias Dinámicas. Lima, Perú.

3. Universidad de San Martín de Porres, Facultad de Medicina Humana, Instituto de Investigación. Lima, Perú.

a. Médico Geriatra.

b. Jefe del Departamento de Medicina Interna. 


\section{INTRODUCCIÓN}

La malnutrición relacionada con el cáncer es un proceso multifactorial donde no solo se afecta la ingesta de alimentos, sino también se incrementa la necesidad de energía y proteínas, disminuyen los estímulos anabólicos, como la actividad física, y se altera el metabolismo en diferentes órganos o tejidos ${ }^{(1)}$.

La pérdida de peso involuntaria es capaz de predecir la supervivencia global, así como, una baja respuesta a la quimioterapia ${ }^{(2,3)}$. La malnutrición y la caquexia son temas relativamente poco investigados en la atención del cáncer, están relacionados con la poca tolerancia al tratamiento oncológico y la menor probabilidad de respuesta, y de manera independiente predicen resultados deficientes ${ }^{(4-6)}$.

El cuestionario Mini Nutritional Assessment (MNA) ofrece una mejor exploración del estado nutricional que la medida de índice de masa corporal y la albúmina ${ }^{(7)}$. En un estudio comparativo de los índices bioquímicos, medidas antropométricas y el MNA, este último se encontró que estaba fuertemente correlacionada con la pérdida de peso inicial en los pacientes con cáncer y se recomienda como una herramienta de evaluación nutricional ${ }^{(8)}$.

El presente estudio tuvo como objetivo evaluar el estado de nutrición como predictor de mortalidad en los adultos mayores con cáncer gástrico, después de un año de seguimiento.

\section{MATERIALES Y MÉTODOS}

Estudio observacional y prospectivo que incluyó 47 pacientes mayores de 60 años con diagnóstico de cáncer gástrico remitido al Servicio de Geriatría del Hospital Nacional Guillermo Almenara, de marzo del 2014 a octubre del 2015.

Para evaluar el estado nutricional se utilizó el Mini Nutritional Assessment Short-Form, de acuerdo a la suma de las puntuaciones obtenidas en cada ítem se clasificaron en estado nutricional adecuado (14-12 puntos); en riesgo de desnutrición (11-8 puntos) y la desnutrición (7-0 puntos) ${ }^{(9)}$. Se considera obesidad para valores de $I M C \geq 30 \mathrm{~kg} / \mathrm{m}^{2}$; sobrepeso entre 27 y $29,9 \mathrm{~kg} / \mathrm{m}^{2}$; normopeso entre 22 y $26,9 \mathrm{~kg} / \mathrm{m}^{2}$; peso insuficiente entre 18,5 y $21,9 \mathrm{~kg} / \mathrm{m}^{2}$ y desnutrición por valores de IMC inferiores a $18,5 \mathrm{~kg} / \mathrm{m}^{2}{ }^{(10)}$. Se debe anotar que la valoración del IMC forma parte del MNA-SF.

Los datos de mortalidad fueron obtenidos del Registro Nacional de Identidad del Perú (RENIEC).

Se procedió con el análisis descriptivo de cada variable de estudio, resumiéndose las de naturaleza cualitativa mediante tablas de frecuencias absolutas y relativas, y las de naturaleza cuantitativa mediante la media y desviación estándar. Con el objeto de conocer la mortalidad tras 12 meses de seguimiento se construyó un modelo de regresión de Cox.

\section{RESULTADOS}

Se incluyeron 47 pacientes con diagnóstico de cáncer gástrico, $33(70,2 \%)$ fueron de sexo masculino, la media de edad fue 75,52 (DE +/ - 6,88) años para los varones y 77 (DE $+/-7,66)$ años para las mujeres. Las demás características de la población se resumen (Tabla 1).

Tabla 1. Características de los adultos mayores con cáncer gástrico

\begin{tabular}{lcc}
\hline & Frecuencia (n) & Porcentaje (\%) \\
\hline Edad (años) & & \\
\hline - Menores de 80 años & 28 & 59,6 \\
- De 80 años a más & 19 & 40,4 \\
\hline Sexo & & \\
- Masculino & 33 & 70,2 \\
- Femenino & 14 & 29,8 \\
Comorbilidad (índice de Charlson) & & \\
- Baja comorbilidad & 34 & 72,3 \\
- Alta comorbilidad & 13 & 27,7 \\
Estado nutricional (IMC) & & \\
- Desnutrición & 3 & 6,4 \\
- Peso insuficiente & 12 & 25,5 \\
- Normopeso & 22 & 46,8 \\
- Sobrepeso & 5 & 8,5 \\
\hline
\end{tabular}


Estado nutricional como predictor de mortalidad en el adulto mayor con cáncer gástrico evaluado en un hospital de referencia

\begin{tabular}{|lcc|} 
& Frecuencia (n) & Porcentaje (\%) \\
\hline Riesgo nutricional (MNA - SF) & & \\
\hline - Estado nutricional normal & 5 & 10,6 \\
- Riesgo de malnutrición & 21 & 44,7 \\
- Desnutrición & 21 & 44,7 \\
Extensión de enfermedad & & \\
- Sin metástasis. & 30 & 63,8 \\
- Con metástasis. & 17 & 36,2 \\
\hline
\end{tabular}

La diferencia del promedio del IMC de los pacientes vivos y los fallecidos fue de 4,47 kilos, y del MNA-SF fue de 2,4 puntos. Las demás variables se resumen (Tabla 2).

Tabla 2. Mortalidad según edad, sexo, estado nutricional y tratamiento. Tiempo de seguimiento: 12 meses

\begin{tabular}{|c|c|c|c|}
\hline & $\begin{array}{l}\text { Vivos } \\
(n=20)\end{array}$ & $\begin{array}{c}\text { Fallecidos } \\
(\mathbf{n}=27)\end{array}$ & $\mathbf{p}$ \\
\hline Edad (media, SD)* & $76,40(7,37)$ & $75,63(6,95)$ & 0,71 \\
\hline Sexo masculino $(n, \%)^{* *}$ & $12(60)$ & $21(77,78)$ & 0,18 \\
\hline Índice de masa corporal (media, SD)* & $26,34(5,12)$ & $21,87(2,58)$ & $<0,05$ \\
\hline MNA - SF (media, SD)* & $9,25(2,09)$ & $6,85(2,26)$ & $<0,05$ \\
\hline Metástasis $(n, \%)^{\star *}$ & $1(8,3)$ & $12(57,1)$ & 0,17 \\
\hline Tratamiento paliativo (n, \%) ${ }^{\star \star}$ (no quimioterapia ni cirugía) & $1(5)$ & $10(37)$ & $<0,05$ \\
\hline Quimioterapia $(\mathrm{n}, \%)^{\star *}$ & $8(40)$ & $5(18,52)$ & 0,10 \\
\hline Cirugía $(n, \%)^{* *}$ & $15(75)$ & $16(59,26)$ & 0,26 \\
\hline Quimioterapia y cirugía $(\mathrm{n}, \%)^{\star *}$ & $4(20)$ & $4(14,8)$ & 0,94 \\
\hline
\end{tabular}

* Prueba de T de Student para igualdad de medias de muestras independientes.

** Prueba de Chi cuadrado de Paerson.

Durante el periodo de seguimiento del estudio, 31 pacientes fueron operados, 13 recibieron quimioterapia, 8 ambos tratamientos. La mortalidad total fue de 57,44 por ciento, de los cuales 21 fueron varones.

Al realizar el análisis de supervivencia, se encontró que la evaluación nutricional mediante el MNA-SF obtuvo significancia estadística $(p<0.05)($ Tabla 3$)$.

Tabla 3. Regresión de Cox, para el análisis de supervivencia a un año. Comparación entre MNA-SF e IMC

\begin{tabular}{|c|c|c|c|c|c|}
\hline & \multirow[t]{2}{*}{$\mathbf{P}$} & \multirow[t]{2}{*}{$\mathrm{HR}^{*}$} & \multirow[t]{2}{*}{ Media } & \multicolumn{2}{|c|}{ IC (95 \%) } \\
\hline & & & & $\begin{array}{l}\text { Límite } \\
\text { inferior }\end{array}$ & $\begin{array}{l}\text { Límite } \\
\text { superior }\end{array}$ \\
\hline Índice de masa corporal $\left(\mathrm{k} / \mathrm{m}^{2}\right)$ & 0,63 & 0,97 & 21,87 & 0,85 & 1,10 \\
\hline MNA - SF & 0,05 & 0,84 & 6,85 & 0,70 & 0,99 \\
\hline
\end{tabular}

* HR: hazard ratio

La media de supervivencia global en meses fue de 5,18 (SE 1,44 IC 95\%) para quienes obtuvieron valores superiores a 7 en el MNA-SF y de 2,93 (SE 0,85 IC $95 \%$ ) en puntuaciones inferiores (Figura 1). 


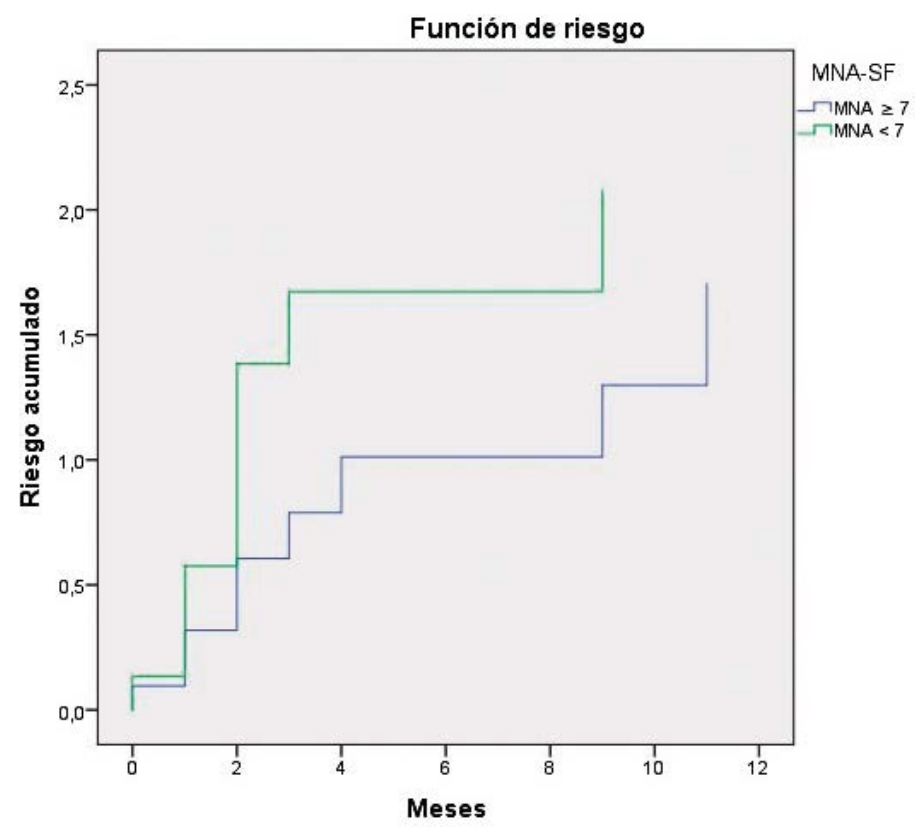

Figura 1. Kaplan-Meyer del riesgo acumulado de mortalidad a 12 meses según el MNA-SF

\section{DISCUSIÓN}

Cada vez más se reconoce la importancia del estado nutricional, y su impacto en la evolución del cáncer gástrico. El estudio demuestra que no solo las comorbilidades, la estirpe celular, la extensión de la enfermedad, el tratamiento oncológico influyen en la mortalidad de los ancianos con este tipo de patología (11-17).

La caquexia y la pérdida de peso involuntaria son las principales consecuencias de los cambios metabólicos de un paciente con cáncer ${ }^{(18,19)}$. Además, comprometen la capacidad de los pacientes para recibir, tolerar y responder a la terapia médica o quirúrgica, y predicen un resultado deficiente independientemente de otros factores de riesgo $(4,20)$.

Solo el 10,6 por ciento de los pacientes evaluados en el presente estudio tuvieron un estado nutricional normal al momento de la evaluación con el MNA-SF, y aproximadamente el 32 por ciento presentó un peso por debajo de lo normal. Se evidenció que los mayores puntajes en el MNA-SF están asociados a menor riesgo de mortalidad tal como lo demostraron otros autores ${ }^{(8,21)}$.

Las limitaciones del estudio fueron el poco número de pacientes evaluados y el corto tiempo de seguimiento los cuales podrían influir en la significancia estadística a favor o en contra del resultado final. Además, existen factores de riesgo adicionales o diferentes que son predictivos de mortalidad como la estirpe tumoral o el régimen de tratamiento, los cuales no fueron incluidos en el estudio.

En conclusión, el estado nutricional deficiente es un factor pronóstico en la mortalidad del cáncer gástrico en personas mayores. El MNA-SF es el instrumento que mejor predice la mortalidad comparada con la medición únicamente del IMC (22).

\section{REFERENCIAS BIBLIOGRÁFICAS}

1. Arends J, Baracos V, Bertz H, Bozzetti F, Calder PC, Deutz NEP, et al. ESPEN expert group recommendations for action against cancer-related malnutrition. Clin Nutr. 2017;36(5):1187-96. Disponible en: http://www.clinicalnutritionjournal.com/ article/S0261-5614(17)30228-5/pdf

2. Donohoe CL, Ryan AM, Reynolds JV. Cancer Cachexia: Mechanisms and Clinical Implications. Gastroenterol Res Pract 2011;2011:13. Disponible en: https://www.hindawi.com/ journals/grp/2011/601434/abs/

3. Stojcev Z, Matysiak K, Duszewski M, Banasiewicz T. The role of dietary nutrition in stomach cancer. Contemp Oncol. 2013;17(4):343-5. Disponible en: https://www.ncbi.nlm.nih. gov/pmc/articles/PMC3934052/

4. Aapro M, Arends J, Bozzetti F, Fearon K, Grunberg SM, Herrstedt J, et al. Early recognition of malnutrition and cachexia in the cancer patient: a position paper of a European School of Oncology Task Force. Ann Oncol. 2014;25(8):14929. Disponible en: https://academic.oup.com/annonc/ article/25/8/1492/271866

5. Fukuda Y, Yamamoto K, Hirao M, Nishikawa K, Maeda S, Haraguchi N, et al. Prevalence of Malnutrition Among Gastric 
Cancer Patients Undergoing Gastrectomy and Optimal Preoperative Nutritional Support for Preventing Surgical Site Infections. Ann Surg Oncol. 2015;22(3):S778-85. Disponible en: https://link.springer.com/article/10.1245/s10434-0154820-9

6. Son Y-G, Kwon IG, Ryu SW. Assessment of nutritional status in laparoscopic gastrectomy for gastric cancer. Transl Gastroenterol Hepatol. 2017;2:85. Diponible en: https:// www.ncbi.nlm.nih.gov/pmc/articles/PMC5676202/

7. Vellas B, Guigoz Y, Garry PJ, Nourhashemi F, Bennahum D, Lauque $S$, et al. The Mini Nutritional Assessment (MNA) and its use in grading the nutritional state of elderly patients. Nutrition. 1999;15(2):116-22. Disponible en: https://www. ncbi.nlm.nih.gov/pubmed/9990575

8. Slaviero KA, Read JA, Clarke SJ, Rivory LP. Baseline nutritional assessment in advanced cancer patients receiving palliative chemotherapy. Nutr Cancer. 2003;46(2):148-57. Disponible en:https://www.tandfonline.com/doi/abs/10.1207/ S15327914NC4602_07

9. Guigoz Y, Vellas B, Garry PJ. Assessing the nutritional status of the elderly: The Mini Nutritional Assessment as part of the geriatric evaluation. Nutr Rev. 1996;54(1 Pt 2):S59-65. Disponible en: https://www.ncbi.nlm.nih.gov/ pubmed/8919685

10. Camina-Martín MA, de Mateo-Silleras B, Malafarina V, LopezMongil R, Niño-Martín V, López-Trigo JA, et al. Valoración del estado nutricional en Geriatría: declaración de consenso del Grupo de Nutrición de la Sociedad Española de Geriatría y Gerontología. Rev Esp Geriatr Gerontol. 2016;51(1):52-7. Disponible en: https://www.sciencedirect.com/science/ article/pii/S0211139X15001341

11. Anderson SE, Minsky BD, Bains M, Hummer A, Kelsen D, Ilson $\mathrm{DH}$. Combined modality chemoradiation in elderly oesophageal cancer patients. Br J Cancer. 2007;96(12):1823-7. Disponible en: https://www.nature.com/articles/6603821

12. Jorgensen TL, Hallas J, Friis S, Herrstedt J. Comorbidity in elderly cancer patients in relation to overall and cancerspecific mortality. $\mathrm{Br} J$ Cancer. 2012;106(7):1353-60. Disponible en: https://www.nature.com/articles/bjc201246

13. Gooiker GA, Dekker JW, Bastiaannet E, van der Geest LG, Merkus JW, van de Velde CJ, et al. Risk factors for excess mortality in the first year after curative surgery for colorectal cancer. Ann Surg Oncol. 2012;19(8):2428-34. Disponible en: https: //link.springer.com/article/10.1245/s10434-012-22946

14. Søgaard M, Thomsen RW, Bossen KS, Sørensen HT, Nørgaard M. The impact of comorbidity on cancer survival: a review. J Clin Epidemiol. 2013;5(Suppl 1):3-29. Disponible en: https:// www.ncbi.nlm.nih.gov/pmc/articles/PMC3820483/

15. Gretschel S, Estevez-Schwarz L, Hunerbein M, Schneider U, Schlag PM. Gastric cancer surgery in elderly patients. World J Surg Oncol. 2006;30(8):1468-74. Disponible en: https://www. ncbi.nlm.nih.gov/pubmed/16850149

16. Mayol-Oltra A, Marti-Obiol R, Lopez-Mozos F, BaguenaRequena G, Ortega-Serrano J. The influence of advanced age on the morbi-mortality of gastric cancer after curative surgery. Rev Esp Enferm Dig. 2013;105(4):1949. Disponible en: https://pdfs.semanticscholar.org/470a/ a4677febe62a79b4a19f979a61397bf8cab9.pdf
17. Mohammadzadeh F, Noorkojuri H, Pourhoseingholi MA, Saadat $S$, Baghestani AR. Predicting the probability of mortality of gastric cancer patients using decision tree. Ir J Med Sci. 2015;184(2):277-84. Disponible en: https://link.springer. com/article/10.1007/s11845-014-1100-9

18. Fearon KC, Voss AC, Hustead DS. Definition of cancer cachexia: effect of weight loss, reduced food intake, and systemic inflammation on functional status and prognosis. Am J Clin Nutr. 2006;83(6):1345-50. Disponible en: https://academic. oup.com/ajcn/article/83/6/1345/4633039

19. Seo KW, Yoon KY. Nutritional assessment and perioperative nutritional support in gastric cancer patients. Korean J Gastroenterol. 2013;61(4):186-90. Disponible en: https: / / synapse.koreamed.org/DOIx.php?id=10.4166/ kjg.2013.61.4.186

20. Steenhagen E, van Vulpen JK, van Hillegersberg R, May $A M$, Siersema PD. Nutrition in peri-operative esophageal cancer management. Expert Rev Gastroenterol Hepatol. 2017;11(7):663-72. Disponible en: https://www.tandfonline. com/doi/abs/10.1080/17474124.2017.1325320

21. Read JA, Crockett N, Volker DH, MacLennan P, Choy ST, Beale $P$, et al. Nutritional assessment in cancer: comparing the Mini-Nutritional Assessment (MNA) with the scored Patient-Generated Subjective Global Assessment (PGSGA). Nutr Cancer. 2005;53(1):51-6. Disponible en: https://www. tandfonline.com/doi/abs/10.1207/s15327914nc5301_6

22. Bourdel-Marchasson I, Diallo A, Bellera C, Blanc-Bisson C, Durrieu J, Germain C, et al. One-Year Mortality in Older Patients with Cancer: Development and External Validation of an MNA-Based Prognostic Score. PLoS ONE. 2016;11(2):e0148523. Disponible en: http://journals.plos. org / plosone $/$ article?id=10.1371/journal. pone. 0148523

Fuentes de financiamiento:

Este artículo ha sido financiado por los autores.

Conflictos de interés:

Los autores declaran no tener ningún conflicto de interés.

\section{Correspondencia:}

Edwin Cesar Cieza Macedo

Dirección: Av. Grau 800 - La Victoria - Lima

Teléfono: 01-324 2983

Correo electrónico: eccm23@gmail.com

Recibido: 17 de enero de 2018

Evaluado: 19 de enero de 2018

Aprobado: 23 de febrero de 2018

(c) La revista. Publicado por Universidad de San Martín de Porres, Perú. (c) $)_{\text {BY }}$ Licencia de Creative Commons Artículo en acceso abierto bajo términos de Licencia Creative Commons Atribución 4.0 Internacional. (http://creativecommons.org/licenses/by/4.0/)

ORCID iDs

Edwin Cieza

Teodoro J. Oscanoa

https: / /orcid.org/0000-0002-8766-1412 https://orcid.org/0000-0001-9379-4767 